\title{
Effect of Scalar Mass in the Absorption and Emission Spectra of Schwarzschild Black Hole
}

\author{
Eylee Jung* and D. K. Park ${ }^{\dagger}$, \\ Department of Physics, Kyungnam University, Masan, 631-701, Korea
}

\begin{abstract}
Following Sanchez's approach we investigate the effect of scalar mass in the absorption and emission problems of $4 d$ Schwarzschild black hole. The absorption cross sections for arbitrary angular momentum of the scalar field are computed numerically in the full range of energy by making use of the analytic near-horizon and asymptotic solutions and their analytic continuations. The scalar mass makes an interesting effect in the low-energy absorption cross section for S-wave. Unlike the massless case, the cross section decreases with increasing energy in the extremely low-energy regime. As a result the universality, i.e. low-energy cross section for S-wave is equal to the horizon area, is broken in the presence of mass. If the scalar mass is larger than a critical mass, the absorption cross section becomes monotonically decreasing function in the entire range of energy. The Hawking emission is also calculated numerically. It turns out that the Planck factor generally suppresses the contribution of higher partial waves except S-wave. The scalar mass in general tends to reduce the emission rate.
\end{abstract}

\footnotetext{
*Email:eylee@kyungnam.ac.kr

†Email:dkpark@hep.kyungnam.ac.kr
} 


\section{INTRODUCTION}

Black hole is an extremely important physical system in which we can test our formulation of quantum gravity and string theories. For example, recent understanding of BekensteinHawking entropy $[1,2]$ based on the microstates $[3,4]$ gives an impetus to recent drastic development of the non-perturbative string theories. The typical phenomenon appearing in black hole physics as a quantum gravity effect is an Hawking radiation $[5,6]$, which states that black hole emits a thermal radiation at Hawking temperature. The Hawking temperature $T_{B H}$ for the non-rotating and chargeless black hole is $T_{B H}=1 / 4 \pi r_{s}$ where $r_{s}$ is an horizon radius. For example, the emission rate of energy in each mode of frequency $k$ and angular momentum $\ell$ can be written as

$$
d H_{\ell}(k)=\frac{\Gamma_{\ell}(k)}{e^{k / T_{B H}} \mp 1} \frac{2 \ell+1}{\pi} k d k
$$

where the upper (lower) sign in the denominator is for boson (fermion). The quantity $\Gamma_{\ell}(k)$ in numerator is an absorption coefficient, which is also named 'greybody' factor. From a viewpoint of quantum mechanical scattering theory, it is related to the scattering amplitude $S_{\ell}(k)$ in the form

$$
\Gamma_{\ell}(k)=1-\left|S_{\ell}(k)\right|^{2}
$$

In usual quantum mechanical systems $S_{\ell}(k)$ is an unitary quantity, i.e. $S_{\ell}=e^{2 i \delta_{\ell}}$, where the real parameter $\delta_{\ell}$ is a phase shift. Thus, $\Gamma_{\ell}(k)$ vanishes in these systems. However, the amplitude $S_{\ell}(k)$ is not in general phase factor in the black hole system [7], which makes the phase shift to be complex, i.e. $\delta_{\ell}=\eta_{\ell}+i \beta_{\ell}$. The non-unitarity of the scattering amplitude might be closely related to the information loss problem of the black hole [8-10] although Hawking stated the information loss puzzle in terms of the quantum pure state and quantum mixed state [8].

Eq.(1.1) indicates that the calculation of the absorption coefficient $\Gamma_{\ell}(k)$ is essential for the computation of the Hawking radiation. As will be shown shortly (see Eq.(2.5) and 
Eq.(4.3)), $\Gamma_{\ell}(k)$ can be straightforwardly computed from the partial absorption cross section $\sigma_{\ell}(k)$. Thus, it is important to compute $\sigma_{\ell}(k)$ strictly as much as possible.

The rough estimation of the absorption and emission rates for the various black holes ${ }^{1}$ were computed long ago [12-15]. Especially Unruh derived in his seminal paper [16] the analytic expression of the low-energy partial absorption cross section for the massive scalar and Dirac particles by matching the near-horizon radial solution and the asymptotic solution via the solution in the intermediate regime. It is instructive to introduce an explicit expression for the partial absorption cross section of Ref. [16] for the massive scalar:

$$
\begin{aligned}
& \left(\sigma_{\ell}\right)_{\text {unruh }}=\frac{\pi}{k^{2} v^{2}}(2 \ell+1) T_{\ell} \\
& T_{\ell}=\frac{\pi(\ell !)^{4} 2^{2 \ell+2}\left(1+v^{2}\right) k^{2 \ell+3} v^{2 \ell}}{(2 \ell !)^{4}(2 \ell+1)^{2}\left[1-\exp \left\{-\pi k\left(1+v^{2}\right) / v\right\}\right]} \prod_{s=1}^{\ell}\left[s^{2}+\left(\frac{k\left(1+v^{2}\right)}{2 v}\right)^{2}\right]
\end{aligned}
$$

where $v=\sqrt{1-m^{2} / k^{2}}$. When Unruh derived Eq.(1.3), he put the horizon radius of the Schwarzschild black hole to be unity.

The most interesting result in Eq.(1.3) is the $S$-wave cross section which is

$$
\left(\sigma_{0}\right)_{\text {unruh }}=\frac{(2 \pi)^{2}\left(1+v^{2}\right) k}{v^{2}\left[1-\exp \left\{-\pi k\left(1+v^{2}\right) / v\right\}\right]} .
$$

Taking $m \rightarrow 0$ limit and assuming $k<<1$, we have $\left(\sigma_{0}\right)_{\text {unruh }} \sim 4 \pi$, which equals to the horizon area. This is an universal property of the low-energy absorption cross section for massless scalar in black hole physics.

Inspired by string theories, the universal property is re-examined few years ago in the arbitrary-dimensional black hole [17] and higher-dimensional objects [18] such as p-brane. More recently, it is also examined whether or not the effect of mass maintains the universal property of the low-energy absorption cross section in the higher-dimensional theories $[19,20]$. Although the mass effect in general does not break the universal property on condition that

\footnotetext{
${ }^{1}$ The Hawking radiation for near-extremal $5 d$ black hole and its implications in the information puzzle are discussed in Ref. [11]
} 
mass is not too large, it is found that the mass-dependence of the cross section is very sensitive to the spacetime property especially, in the near-horizon regime.

While the low-energy behavior of the absorption and emission in $4 d$ black hole and higherdimensional objects were extensively examined, the behavior in different range of energy is not sufficiently examined. Fortunately, for D3-brane system in Born-Infeld theories [21,22], however, the wave equation is represented in terms of the modified Mathieu equation. Using the various properties of the equation it is possible to examine the absorption cross section in high-energy domain as well as low-energy domain [23-27]. However, fairly speaking, it seems to be far away to understand the absorption and emission spectra in the full range of energy in the general higher-dimensional systems.

For $4 d$ black hole system the full absorption spectrum of Schwarzschild black hole for the massless scalar was computed long ago $[28,29]$ by making use of the analytical series expressions of near-horizon and asymptotic solutions and their analytic continuations. In fact, these expressions and their mathematical properties were examined in detail by Persides [30-32]. In Ref. [29] Sanchez matched these two analytical solutions via analytic continuation, which enables to compute the absorption and emission spectra in the full range of energy.

In this paper we would like to study the effect of mass in the absorption and emission spectra of black hole in the full range of energy. This seems to be important due to the fact that most matters have their own masses. However, the effect of mass can be negligible in the realistic situation because matter's mass is in general extremely small compared with black hole's mass. Our particular interest is to check whether the mass effect maintains the universal property of low-energy absorption cross section or not. Also, it is of interest to check how the mass effect changes the global properties of the absorption and emission spectra.

The numerical calculation shows that the absorption cross section for massive scalar case is in general larger than that for massless case. In particular, for the S-wave the introduction of mass makes an unexpected effect in the low-energy regime of the partial cross section. In 
the extremely low-energy regime the partial cross section decreases with increasing energy unlike the case of massless scalar field. This indicates that the universality is generally broken due to the scalar mass. As Sanchez found in the massless case [28,29], the total absorption cross section has an wiggly characteristic which implies that each partial absorption cross section has a peak in different energy scale. However, the total emission rate does not have the oscillatory pattern, which indicates that the Planck factor in general suppresses the contribution of the higher partial waves. It turns out that the scalar mass generally reduces the emission rate.

The paper is organized as follows. In section II we will describe the results for the massless case briefly without any calculation. In the same section we will comment the possible physical mechanism which explains the results roughly. In section III the wave equation for the massive scalar is solved to derive the analytic solutions in the near-horizon and asymptotic regimes. These two solutions are matched with each other by making use of the analytic continuation in section IV. The numerical calculation is performed in the same section to examine the effect of the scalar mass in the emission and absorption spectra of black hole. In section $\mathrm{V}$ a brief conclusion is given. In appendix the Wronskians for the analytic solutions are explicitly calculated.

\section{BRIEF DESCRIPTION OF RESULTS FOR MASSLESS CASE}

In this section we will comment briefly the results of the absorption and emission prob-

lems for the massless scalar case. The minimally coupled scalar field equation $\square \Phi=0$ in the background of Schwarzschild spacetime, whose metric is

$$
g_{\mu \nu}=\operatorname{diag}\left[\left(1-\frac{r_{s}}{r}\right),-\left(1-\frac{r_{s}}{r}\right)^{-1},-r^{2},-r^{2} \sin ^{2} \theta\right]
$$

where $r_{s}$ is an horizon radius, reduces to the following radial wave equation

$$
x\left(x-x_{s}\right)^{2} \frac{d^{2} R_{\ell}}{d x^{2}}+\left(x-x_{s}\right)\left(2 x-x_{s}\right) \frac{d R_{\ell}}{d x}+\left[x^{3}-\ell(\ell+1)\left(x-x_{s}\right)\right] R_{\ell}=0
$$


where $\Phi=R_{\ell}(r) Y_{\ell, m}(\theta, \phi) e^{-i k t}, x=k r$, and $x_{s}=k r_{s}$. This radial equation was analytically solved in Ref. [30] at near-horizon and asymptotic regions separately as a series form and the mathematical properties of them were analyzed in Ref. [31,32]. These two solutions are matched in Ref. [28] by the analytic continuation which makes a common domain of convergence for the solutions. As a result, one can compute the absorption and emission spectra numerically. We do not want to repeat the calculation here. What we want to do in this section is just a brief description of the results and possible mechanisms for explaining why these results come out.

Fig. 1 shows the $k$-dependence of the partial absorption cross section $\sigma_{\ell}$ for $\ell=0,1,2$ in the full range of $k$ when $r_{s}=1$. The global shape of $\sigma_{\ell}$ exhibits an increasing and decreasing patterns at small $k$ and large $k$ respectively, and each $\sigma_{\ell}$ has a unique global maximum at $k=k_{\ell}^{*}$. This can be understood as following. If we use a "tortoise" coordinate $x_{*}=x+x_{s} \ln \left(x / x_{s}-1\right)$, the radial equation (2.2) is changed into the usual Schrödinger form as following:

$$
-\frac{d^{2} \psi_{\ell}}{d r_{*}^{2}}+V_{e f f}^{(0)}\left(r_{*}\right) \psi_{\ell}=k^{2} \psi_{\ell}
$$

where $\psi_{\ell}=x R_{\ell}, x_{*}=k r_{*}$ and

$$
V_{e f f}^{(0)}\left(r_{*}\right)=\left(1-\frac{r_{s}}{r}\right)\left[\frac{r_{s}}{r^{3}}+\frac{\ell(\ell+1)}{r^{2}}\right] .
$$

Thus, $V_{e f f}^{(0)}$ and $k^{2}$ play roles of quantum mechanical potential and energy eigenvalue respectively. The potential $V_{e f f}^{(0)}$ is plotted in Fig. 2 for $l=0,1$, and 2 with respect to $r_{*}$. The overall shape of $V_{e f f}^{(0)}$ is a potential barrier which separates the near-horizon and asymptotic regions, and the barrier height is denoted by $\omega_{\ell}^{2}$. The numerical result of $k_{\ell}^{*}$ and $\omega_{\ell}$ are given at Table I.

Table I

\begin{tabular}{|l|l|l|l|l|l|}
\hline \hline & $\ell=0$ & $\ell=1$ & $\ell=2$ & $\ell=3$ & $\ell=4$ \\
\hline$k_{\ell}^{*}$ & 0.239 & 0.687 & 1.099 & 1.503 & 1.903 \\
\hline$\omega_{\ell}$ & 0.325 & 0.630 & 0.994 & 1.370 & 1.750 \\
\hline \hline
\end{tabular}


The Table shows $k_{\ell}^{*}>\omega_{\ell}$ for nonzero $\ell$. This can be understood from the fact that $\sigma_{\ell}$ is proportional to the transmission coefficient (or absorption coefficient) and the maximum transmission coefficient generally occurs when energy is higher than potential barrier height. This fact also roughly explains the increasing behavior of $\sigma_{\ell}$ in low-energy region. The decreasing behavior of $\sigma_{\ell}$ in high-energy region can be explained from the relation of $\sigma_{\ell}(k)$ with $\Gamma_{\ell}(k)$. For massless case this relation is given by

$$
\sigma_{\ell}(k)=\frac{\pi}{k^{2} r_{s}^{2}}(2 \ell+1) \Gamma_{\ell}(k) .
$$

Since $\Gamma_{\ell}(k)$ goes to unity at most in high-energy region, we have $\sigma_{\ell}(k) \sim 1 / k^{2}$, which explains the decreasing behavior of $\sigma_{\ell}$ in the same region. The fact $\omega_{0}>k_{0}^{*}$ may occur because of the competition between $k^{2}$ in denominator and $\Gamma_{0}(k)$ in numerator of Eq.(2.5).

Fig. 1 also indicates $\sigma_{0}(k=0)=4 \pi$, which is an universal property of low-energy absorption cross section for S-wave. The fact of $\sigma_{0}(k=0) \neq 0$ and $\sigma_{\ell}(k=0)=0$ for nonzero $\ell$ implies that $\Gamma_{0}(k) \sim k^{2}$ and $\Gamma_{\ell}(k) \sim k^{\alpha}$ with $\alpha>2$ at low-energy region. This is in good agreement with Starobinsky's formula [12,28]

$$
\Gamma_{\ell} \sim \frac{(\ell !)^{6}}{(2 \ell !)^{2}[(2 \ell+1) !]^{2}}\left(2 k r_{s}\right)^{2 \ell+2}
$$

at low- $k$ regime. The results of Unruh, $\left(\sigma_{\ell}\right)_{\text {unruh }}$ in Eq.(1.3) is plotted by red lines in Fig. 1. Thus the cross sections $\left(\sigma_{\ell}\right)_{\text {unruh }}$ derived by Unruh in Ref. [16] are correct only in the extremely low-energy regime.

The black line in Fig. 3 is a plot of $k$-dependence of total absorption cross section, i.e. $\sigma_{\text {tot }}=\sum_{\ell} \sigma_{\ell}$. The wiggly pattern of $\sigma_{\text {tot }}$ implies the wave-black hole interaction in the nonhermitian effective potential [7]. This also indicates that each partial cross section has a peak in different value of $k$. Similar oscillatory behavior also appears in the total absorption cross section of $D 3$-brane [26]. The limiting value at large- $k$ region is $27 \pi / 4 \sim 21.2$. Based on this limiting value Sanchez made an formula for $\sigma_{\text {tot }}$ :

$$
\sigma_{\text {tot }}(k)=\frac{27 \pi}{4}-\sqrt{2} \frac{\sin (\sqrt{2 \pi} \pi k)}{k} .
$$


The black line in Fig. 4 is a plot of $k$-dependence of emission rate, which is computed by

$$
\begin{array}{r}
\frac{d H}{d k}(k)=\sum_{\ell=0}^{\infty} \frac{d H_{\ell}}{d k}(k) \\
\frac{d H_{\ell}}{d k}(k)=\frac{k^{3} r_{s}^{2} \sigma_{\ell}}{\pi^{2}\left[e^{4 \pi k r_{s}}-1\right]} .
\end{array}
$$

The oscillatory pattern of $\sigma_{t o t}$ disappears in the emission rate because the S-wave contribution predominates in Hawking radiation. The rapidly decrease of the Planck factor for $k>1$ suppresses the contribution of the higher partial waves.

\section{ANALYTIC SOLUTIONS FOR THE MASSIVE SCALAR CASE}

In this section we will derive the analytic solution for massive scalar field coupled to the Schwarzschild background in the near-horizon and asymptotic regimes. The minimally coupled field equation $\left(\square+m^{2}\right) \Phi=0$ in the Schwarzschild spacetime (2.1) provides a following radial equation

$$
\begin{aligned}
x(x- & \left.x_{s}\right)^{2} \frac{d^{2} R_{\ell}}{d x^{2}}+\left(x-x_{s}\right)\left(2 x-x_{s}\right) \frac{d R_{\ell}}{d x} \\
& +\left[x^{3}-\ell(\ell+1)\left(x-x_{s}\right)+\frac{m^{2}}{k^{2} v^{2}} x_{s} x^{2}\right] R_{\ell}=0
\end{aligned}
$$

where $v=\sqrt{1-m^{2} / k^{2}}, x \equiv k v r$ and $x_{s}=k v r_{s}$. Of course, Eq.(3.1) simply reduces to Eq.(2.2) when $m=0$. Unlike Eq.(2.2), however, Eq.(3.1) involves an explicit $m$-dependence, which makes a situation more complicate.

At this stage it is worthwhile noting the meaning of $r_{s}=1$, which we use frequently through a paper. Since the Compton wavelength is inversely proportional to the mass, $m \sim 1$ in this unit corresponds to such a mass of the field when its Compton wavelength is order of the gravitational radius.

Before solving Eq.(3.1) it is instructive to consider the effective potential. Letting $x_{*}=$ $x+x_{s} \ln \left(x / x_{s}-1\right)$ and $\psi_{\ell}=x R_{\ell}$ make Eq.(3.1) to be 


$$
\begin{gathered}
-\frac{d^{2} \psi_{\ell}}{d r_{*}^{2}}+V_{e f f, \ell} \psi_{\ell}=k^{2} v^{2} \psi_{\ell} \\
V_{e f f, \ell}\left(r_{*}\right)=\left(1-\frac{r_{s}}{r}\right)\left[\frac{r_{s}}{r^{3}}+\frac{\ell(\ell+1)}{r^{2}}\right]-\frac{m^{2} r_{s}}{r}
\end{gathered}
$$

where $r_{*}=x_{*} / k v$. Thus $V_{e f f, \ell}$ and $k^{2} v^{2}$ play same roles with a quantum mechanical potential and energy eigenvalue.

The $r_{*}$-dependence of $V_{e f f, \ell}$ is plotted in Fig. 5 for $\ell=0,1$ and 2. The mass term in $V_{\text {eff, } \ell}$ generally lowers the barrier height. Thus, there exists an critical mass $m_{c}$ for each $\ell$ such that the barrier height is lower than the asymptotic value of $V_{e f f, \ell}$. The numerical calculation shows $m_{c}=0.385$ for $\ell=0, m_{c}=0.795$ for $\ell=1$, and $m_{c}=1.277$ for $\ell=2$ when $r_{s}=1$. Thus if $m>m_{c}$ for each $\ell$, the absorption coefficient $\Gamma_{\ell}(k)$ becomes large, which should enhance the absorption cross section in the low-energy domain $k \approx m$. Since, furthermore, the mass term in $V_{e f f, \ell}$ makes the near-horizon regime to be more stable than the asymptotic region, $\Gamma_{\ell}(k)$ may become non-zero at $k=m \neq 0$, which also enhances the absorption cross section in the low-energy domain.

Following Ref. [30] it is straight forward to derive the analytic solutions $\mathcal{G}_{\ell}\left(x, x_{s}\right)$ in the near-horizon region and $\mathcal{F}_{\ell( \pm)}\left(x, x_{s}\right)$ in the asymptotic region as a series form:

$$
\begin{array}{r}
\mathcal{G}_{\ell}\left(x, x_{s}\right)=e^{-i \frac{x_{s}}{v} \ln \left|x-x_{s}\right|} \sum_{n=0}^{\infty} d_{\ell, n}\left(x-x_{s}\right)^{n} \\
\mathcal{F}_{\ell( \pm)}\left(x, x_{s}\right)=( \pm i)^{\ell+1} e^{\mp i\left(x+\frac{x_{s}}{v} \ln \left|x-x_{s}\right|\right)} \sum_{n=0}^{\infty} \tau_{n( \pm)} x^{-(n+1)}
\end{array}
$$

The coefficients $d_{\ell, n}$ and $\tau_{n( \pm)}$ satisfy the following recurrence relations:

$$
\begin{array}{r}
{\left[\left(n-i \frac{x_{s}}{v}\right)^{2}+x_{s}^{2}\left(1+\frac{m^{2}}{k^{2} v^{2}}\right)\right] x_{s} d_{\ell, n}+\left[\left(n-i \frac{x_{s}}{v}-1\right)\left(n-i \frac{x_{s}}{v}\right)-\ell(\ell+1)\right.} \\
\left.+x_{s}^{2}\left(3+\frac{2 m^{2}}{k^{2} v^{2}}\right)\right] d_{\ell, n-1}+\left(3+\frac{m^{2}}{k^{2} v^{2}}\right) x_{s} d_{\ell, n-2}+d_{\ell, n-3}=0 \\
{\left[ \pm 2 i n+x_{s}\left(\frac{1}{v}-1\right)^{2}\right] \tau_{n( \pm)}-\left[(\ell+n)(\ell-n+1) \mp i x_{s}\left(\frac{1}{v}-1\right)\right] \tau_{(n-1)( \pm)}} \\
-(n-1)^{2} x_{s} \tau_{(n-2)( \pm)}=0
\end{array}
$$

where $\tau_{0( \pm)}=1$. We will show shortly that the coefficient $d_{\ell, 0}$ is an important quantity for the study of absorption problem from the viewpoint of quantum mechanical scattering 
theory. In Eq.(3.3) we choosed only ingoing wave in near-horizon solution and ingoing and outcoming waves in asymptotic solution. It is interesting to note $\mathcal{F}_{\ell( \pm)}^{*}=\mathcal{F}_{\ell(\mp)}$, which comes from the real nature of Eq.(3.1). In the appendix we will show the Wronskians become

$$
\begin{aligned}
& W\left[\mathcal{G}_{\ell}^{*}\left(x, x_{s}\right), \mathcal{G}_{\ell}\left(x, x_{s}\right)\right]_{x} \equiv \mathcal{G}_{\ell}^{*} \partial_{x} \mathcal{G}_{\ell}-\mathcal{G}_{\ell} \partial_{x} \mathcal{G}_{\ell}^{*}=-\frac{2 i\left|g_{\ell}\right|^{2} x_{s}^{2}}{v x\left(x-x_{s}\right)} \\
& W\left[\mathcal{F}_{\ell(+)}\left(x, x_{s}\right), \mathcal{F}_{\ell(-)}\left(x, x_{s}\right)\right]_{x} \equiv \mathcal{F}_{\ell(+)} \partial_{x} \mathcal{F}_{\ell(-)}-\mathcal{F}_{\ell(-)} \partial_{x} \mathcal{F}_{\ell(+)}=\frac{2 i}{x\left(x-x_{s}\right)}
\end{aligned}
$$

where $g_{\ell} \equiv d_{\ell, 0}$.

Now, we would like to show how the coefficient $g_{\ell}$ is related to the partial scattering amplitude. From the viewpoint of quantum mechanical scattering theory the real scattering solution $R_{\ell}\left(x, x_{s}\right)$ of Eq.(3.1) should be only ingoing wave at the near-horizon region and mixture of ingoing and outcoming waves at the asymptotic region as following:

$$
\begin{aligned}
& R_{\ell} \stackrel{x \rightarrow x_{s}}{\sim} g_{\ell}\left(x-x_{s}\right)^{-i \frac{x_{s}}{v}}\left[1+O\left(x-x_{s}\right)\right] \\
& R_{\ell} \stackrel{x \rightarrow \infty}{\sim} i^{\ell+1} \frac{2 \ell+1}{2 x}\left[e^{-i\left(x+\frac{x_{s}}{v} \ln \left|x-x_{s}\right|\right)}-(-1)^{\ell} S_{\ell}\left(x_{s}\right) e^{i\left(x+\frac{x_{s}}{v} \ln \left|x-x_{s}\right|\right)}\right]+O\left(\frac{1}{x^{2}}\right)
\end{aligned}
$$

where $S_{\ell}$ is a partial scattering amplitude. If we define a phase shift $\delta_{\ell}$ as $S_{\ell} \equiv e^{2 i \delta_{\ell}}$, the second equation of Eq.(3.6) can be written as

$$
R_{\ell} \stackrel{x \rightarrow \infty}{\sim} \frac{2 \ell+1}{x} e^{i \delta_{\ell}} \sin \left[x+\frac{x_{s}}{v} \ln \left|x-x_{s}\right|-\frac{\pi}{2} \ell+\delta_{\ell}\right]+O\left(\frac{1}{x^{2}}\right)
$$

Next let us consider the Wronskian $W \equiv R_{\ell}^{*} \partial_{x} R_{\ell}-R_{\ell} \partial_{x} R_{\ell}^{*}$. Although we do not know the exact expression of $R_{\ell}$ in the full range of $x$, it is enough to use the near-horizon solution given in Eq.(3.6) for the computation of $W$, which yields $W=-2 i\left|g_{\ell}\right|^{2} x_{s}^{2} / v x(x-$ $x_{s}$ ). Thus same value should be obtained if we use the asymptotic solution (3.7) for the computation of $W$. If, however, the phase shift $\delta_{\ell}$ is real, $W$ becomes zero at the leading order which makes a contradiction. Thus we should assume that $\delta_{\ell}$ is complex, i.e. $\delta_{\ell}=$ $\eta_{\ell}+i \beta_{\ell}$. Then the Wronskian $W$ computed by the asymptotic solution becomes $W=$ $-i(2 \ell+1)^{2} \sinh 2 \beta_{\ell} e^{-2 \beta_{\ell}} / x\left(x-x_{s}\right)$. Therefore, equating those Wronskians gives a relation

$$
\left|g_{\ell}\right|^{2}=\frac{v\left(\ell+\frac{1}{2}\right)^{2}}{x_{s}^{2}}\left(1-e^{-4 \beta_{\ell}}\right) .
$$


As a result we can obtain $g_{\ell}$ from the partial scattering amplitude or vice versa.

Now, we would like to discuss how $g_{\ell}$ can be computed by matching the asymptotic and near-horizon solutions with each other. For this it is convenient [28] to introduce a new wave solution $\varphi_{\ell}\left(x, x_{s}\right)$, which differs from $R_{\ell}\left(x, x_{s}\right)$ in its normalization. It is normalized in such a way that

$$
\varphi_{\ell}\left(x, x_{s}\right) \stackrel{x \rightarrow x_{s}}{\sim}\left(x-x_{s}\right)^{-i \frac{x_{s}}{v}}\left[1+O\left(x-x_{s}\right)\right]
$$

Since $\mathcal{F}_{\ell( \pm)}\left(x, x_{s}\right)$ in Eq.(3.3) are two linearly independent solutions of the radial equation (3.1), one may write $\varphi_{\ell}$ as a linear combination of them:

$$
\varphi_{\ell}\left(x, x_{s}\right)=f_{\ell}^{(-)}\left(x_{s}\right) \mathcal{F}_{\ell(+)}\left(x, x_{s}\right)+f_{\ell}^{(+)}\left(x_{s}\right) \mathcal{F}_{\ell(-)}\left(x, x_{s}\right)
$$

where the coefficients $f_{\ell}^{( \pm)}\left(x_{s}\right)$ are called the jost functions. Eq.(3.5) enables us to compute $f_{\ell}^{( \pm)}$if we know $\varphi_{\ell}$ as following:

$$
f_{\ell}^{( \pm)}= \pm \frac{x\left(x-x_{s}\right)}{2 i} W\left[\mathcal{F}_{\ell( \pm)}, \varphi_{\ell}\right]_{x}= \pm \frac{k v r\left(r-r_{s}\right)}{2 i} W\left[\mathcal{F}_{\ell( \pm)}, \varphi_{\ell}\right]_{r}
$$

where $W\left[f_{1}, f_{2}\right]_{r} \equiv f_{1} \partial_{r} f_{2}-f_{2} \partial_{r} f_{1}$. Inserting the explicit form of $\mathcal{F}_{\ell( \pm)}$ given in Eq.(3.3) and comparing it with the asymptotic expression of $R_{\ell}$ in Eq.(3.6), one can easily derive the following two relations

$$
S_{\ell}\left(x_{s}\right)=\frac{f_{\ell}^{(+)}\left(x_{s}\right)}{f_{\ell}^{(-)}\left(x_{s}\right)}
$$

and

$$
g_{\ell}\left(x_{s}\right)=\frac{\ell+\frac{1}{2}}{f_{\ell}^{(-)}\left(x_{s}\right)} .
$$

Inserting (3.13) into (3.8), therefore, yields

$$
1-\left|S_{\ell}\left(x_{s}\right)\right|^{2} \equiv 1-e^{-4 \beta_{\ell}}=\frac{x^{2}}{v\left|f_{\ell}^{(-)}\left(x_{s}\right)\right|^{2}} .
$$

The partial absorption cross section $\sigma_{\ell}$ is defined in terms of $g_{\ell}\left(x_{s}\right)$ as [7]

$$
\sigma_{\ell}=4 \pi \frac{\left|g_{\ell}\left(x_{s}\right)\right|^{2}}{2 \ell+1}
$$


Combining (3.8), (3.13) and (3.15) we can derive

$$
\sigma_{\ell}=\frac{\pi v}{x_{s}^{2}}(2 \ell+1)\left(1-e^{-4 \beta_{\ell}}\right)=\frac{\pi(2 \ell+1)}{\left|f_{\ell}^{(-)}\right|^{2}} .
$$

Thus one can compute all physical quantities such as $\beta_{\ell}, S_{\ell}$ and $\sigma_{\ell}$ from the jost functions $f_{\ell}^{( \pm)}$

\section{NUMERICAL CALCULATION}

In this section we would like to discuss the effect of scalar mass in the absorption and emission problems. For this we should compute the jost functions $f_{\ell}^{( \pm)}\left(x_{s}\right)$ defined in Eq.(3.10) numerically. Once $f_{\ell}^{( \pm)}\left(x_{s}\right)$ are computed, the partial absorption cross section $\sigma_{\ell}$ is straightforwardly computed by Eq.(3.16).

The computational procedure is as following. Firstly we should note that $\varphi_{\ell}\left(x, x_{s}\right)$ defined in Eq.(3.10) can be expressed as $\mathcal{G}_{\ell}\left(x, x_{s}\right)$ in Eq.(3.3) if we assume $d_{\ell, 0}=1$, i.e. $\varphi_{\ell}\left(x, x_{s}\right)=\left.\mathcal{G}_{\ell}\left(x, x_{s}\right)\right|_{d_{\ell, 0}=1}$. This is a convergent expansion around the near-horizon region. Of course, the other expression of $\varphi_{\ell}\left(x, x_{s}\right)$ in Eq.(3.10) is a convergent expansion around the asymptotic region. Since, however, the domains of convergence for these two different expressions are different from each other, we can not use them directly for the computation of $f_{\ell}^{( \pm)}\left(x_{s}\right)$. In other words, we need two expressions which have common domain of convergence. This is achieved by analytic continuation.

In other to perform the analytic continuation we need a solution of the radial equation (3.1), which is a power-series expansion in the neighborhood of an arbitrary point $x=b$. Indeed such a solution can be obtained as following:

$$
R(x)=e^{-i \frac{x_{s}}{v} \ln \left|x-x_{s}\right|} \sum_{n=0}^{\infty} C_{n}(x-b)^{n}
$$

where $C_{n}$ satisfies the recurrence relation

$$
\begin{aligned}
& b y^{2}(n+1)(n+2) C_{n+2}+y(n+1)\left[y(n+1)+b\left(2 n+1-2 i \frac{x_{s}}{v}\right)\right] C_{n+1} \\
& +\left[(b+2 y) n^{2}+\left\{y-2 i \frac{x_{s}}{v}(y+b)\right\} n+b\left(b^{2}-\frac{x_{s}^{2}}{v^{2}}\right)-y\left\{\ell(\ell+1)+i \frac{x_{s}}{v}\right\}+\frac{m^{2} b^{2} x_{s}}{k^{2} v^{2}}\right] C_{n}
\end{aligned}
$$




$$
\begin{aligned}
+\left[(n-1)\left(n-2 i \frac{x_{s}}{v}\right)+3 b^{2}-\ell(\ell+1)+i \frac{x_{s}}{v}\right. & \left.\left(i \frac{x_{s}}{v}-1\right)+\frac{2 m^{2} b x_{s}}{k^{2} v^{2}}\right] C_{n-1} \\
& +\left(3 b+\frac{m^{2} x_{s}}{k^{2} v^{2}}\right) C_{n-2}+C_{n-3}=0
\end{aligned}
$$

and $y=b-x_{s}$. Thus one can compute in principle all the $C_{n}$ 's $(n \geq 2)$ in terms of $C_{0}$ and $C_{1}$, which are also expressed in terms of $R(b)$ and $\partial_{x} R(b)$. This analytic continuation should be repeated from $\varphi_{\ell}\left(x, x_{s}\right)=\left.\mathcal{G}_{\ell}\left(x, x_{s}\right)\right|_{d_{\ell, 0}=1}$ to raise the domain of convergence by using (4.1) and (4.2). Of course, one can apply a similar procedure to $\varphi_{\ell}\left(x, x_{s}\right)$ in Eq.(3.10) to lower the domain of convergence. We made a computer program such that the number of analytic continuation $N$ is dependent on $k$. For example in the low-energy region $(k \sim m)$, $N$ becomes large because the domains of convergence for each solution are too far. Once we make two solutions which have common domains of convergence, the jost functions $f_{\ell}^{( \pm)}$can be computed by Eq.(3.11).

Fig. 6 show the $k$-dependence of $\beta_{\ell}$ for $\ell=0,1,2$ and various $m$ when $r_{s}=1^{2}$. As in the case of massless scalar $\beta_{\ell}$ is a monotonically increasing function with respect to $k$ for the massive case. However, the scalar mass generally decreases the increasing rate of $\beta_{\ell}$. Since $\beta_{\ell}$ becomes smaller when $m$ becomes larger for fixed $k$ and $\ell$, the factor $v / x_{s}^{2}$ in Eq.(3.16) increases and $1-e^{-4 \beta_{\ell}}$ decreases. Thus, the partial cross section $\sigma_{\ell}$ is determined by competition of these two factors.

Fig. 7 shows the numerical results of $k$-dependence of $\sigma_{\ell}$ for $\ell=0,1$, and 2 when $r_{s}=1$. Fig. 7a shows that the scalar mass tends to increase the absorption cross section for S-wave. This means the factor $v / x_{s}^{2}$ in Eq.(3.16) predominates the factor $1-e^{-4 \beta_{\ell}}$ in the entire range of $k$. Fig. $7 \mathrm{~b}$ and Fig. $7 \mathrm{c}$ show that the factor $1-e^{-4 \beta_{\ell}}$ is superior in the low-energy regime for nonzero $\ell$ if the scalar mass is not too large.

\footnotetext{
${ }^{2}$ The unit $r_{s}=1$, which we used in the plots of most figures, implies that the black hole's mass is $1 / 2$ in the Planck unit. Thus, the comparability of the field's mass to the Planckian mass in the figures represents pure theoretical interest.
} 
The most interesting behavior appears at Fig. 7a which is a plot of $\sigma_{\ell=0}$. For $m=0$ the low-energy absorption cross section exhibits the manifest universality, i.e. $\sigma_{0} \sim 4 \pi$. However for the massive case this universality seems to be broken. The low-energy region is distinguished by two regions on the condition of $m<m_{c}$. In the extremely low-energy region the absorption cross section $\sigma_{0}$ is rapidly decreasing with increase of $k$. In the remaining region $\sigma_{0}$ increases with increase of $k$ as usual massless case. As a result, $\sigma_{\ell}$ has a local minimum in the low-energy regime. When, however, the scalar mass $m$ approaches to the critical mass $m_{c} \sim 0.385$, this kind of distinction in the low-energy regime disappears and the absorption cross section becomes monotonically decreasing function in the full range of $k$. This seems to be obvious because the potential barrier, which separates the near-horizon region from the asymptotic region, is any more meaningless for $m>m_{c}$. The effect of scalar mass in $\sigma_{1}$ and $\sigma_{2}$ is shown in Fig. 7b and Fig. 7c. The cross section $\sigma_{\ell}$ with $\ell \neq 0$ seems to exhibit a similar behavior when the scalar mass approaches to the critical mass. However, no distinction in the low-energy regime seems to occur even if $m \ll m_{c}$. Even in this case the absorption cross section is increasing function in the low-energy regime with respect to $k$.

Fig. 8 shows the mass-dependence of S-wave absorption cross section in the low-energy limit $(k \sim m)$. In the real calculation we choosed $k=m+0.004$ and $r_{s}=1$. This figure shows how the universality of the low-energy absorption cross section is broken due to the effect of scalar mass. Fig. 8 indicates that the universality is roughly preserved when $m<0.02$. However the large mass manifestly breaks the universality, which is also consistent with Fig. 7a. The red line in Fig. 8 is a plot of $m$-dependence of $\left(\sigma_{0}\right)_{\text {unruh }}$, which coincides with $\sigma_{0}$ in the neighborhood of the massless region.

Fig. 3 is a plot of total absorption cross section $\sigma_{\text {tot }}$ for various $m$. In the low-energy region the total absorption cross section increases with increase of $m$, which is also evident from Fig. 7. The divergence of the total cross section in the low-energy regime can be understood easily from Eq.(1.4). Of course, the massless limit $(v=1$ and $k \approx 0)$ of $\left(\sigma_{0}\right)_{\text {unruh }}$ is $4 \pi$ as expected. In the massive case, however, the low-energy $\operatorname{limit}(v \approx 0$ and $k \approx m)$ of 
it is $4 \pi^{2} m / v^{2}$, which goes to infinity. Thus, total cross section should go to infinity at this regime. In high-energy region $\sigma_{\text {tot }}$ becomes $m$-independent and approaches to the massless limit $27 \pi / 4$. This fact reflects an approximate conformal symmetry in the high-energy limit [33].

Finally we would like to discuss the effect of scalar mass in the emission problem. For the massive scalar case the relation between the partial absorption cross section $\sigma_{\ell}$ and the absorption coefficient $\Gamma_{\ell}$ is given by [16]

$$
\Gamma_{\ell}(k)=\frac{k^{2} v^{2} r_{s}^{2}}{\pi(2 \ell+1)} \sigma_{\ell}(k) .
$$

Thus the difference from the massless case is a factor $v^{2}$ in the numerator. Combining Eq.(1.1) and (4.3) makes the emission rate to be

$$
\frac{d H_{\ell}}{d k}=\frac{k^{3} v^{2} r_{s}^{2} \sigma_{\ell}}{\pi^{2}\left(e^{4 \pi k r_{s}}-1\right)}
$$

The total emission rate times $10^{4}$ is plotted in Fig. 4 for various $m$. As in the massless case the oscillatory pattern disappears in the emission rate because of dominance of the $\mathrm{S}$-wave contribution. It is interesting to note that in contrast to the absorption case the emission rate decreases with increase of $m$ because the factor $v^{2}$ in Eq.(4.4) reduces the partial emission rate. The Planck factor seems to suppress the contribution of higher partial wave even in the masive case.

\section{CONCLUSION}

The effect of scalar field mass is examined in the absorption and emission problems of the $4 d$ Schwarzschild black hole in the entire range of energy. The most interesting feature may occur in the low-energy absorption cross section for S-wave (see Fig. 7a). The effect of mass seems to divide the low-energy region into two parts. In the extremely low-energy

region (region I) the $\mathrm{S}$-wave absorption cross section $\sigma_{0}$ rapidly decreases with increase of energy. The decreasing rate becomes larger and larger as the scalar field becomes heavier 
and heavier. In the moderately low-energy region (region II) $\sigma_{0}$ increases with increase of energy as usual massless case. This type of behavior does not appear in massless case, whose $\sigma_{0}$ has only region II in the low-energy regime. When the scalar mass is less than the critical mass $m_{c} \sim 0.385$, where the barrier height of the effective potential (3.2) becomes zero, both regions co-exist, but region I extends and region II shrinks with increasing $m$. When $m$ approaches $m_{c}$, region II finally disappears and the S-wave cross section becomes the monotonically decreasing function in the full-range of energy.

Another interesting feature in the absorption problem is a fact that the heavy scalar particle generally breaks the universal property, i.e. $\lim _{k \rightarrow 0} \sigma_{0} \sim S$, where $S$ is an area of the horizon. The numerical calculation indicates that this universality is roughly preserved when $m<0.02$ (see Fig. 8).

There appears a wiggly pattern in the total absorption cross section as in the massless case [28,29], which indicates a wave-black hole interaction (see Fig. 3). This oscillatory pattern also indicates that each partial absorption cross section has a peak in the different point of energy. Athough the total absorption cross section for massive case is larger than that for massless case in the low-energy region, both approach to $27 \pi / 4$ in the high-energy limit, which reflects an approximate conformal symmetry.

The emission rate does not show the wiggly pattern (see Fig. 4) because the Planck factor usually suppresses the contribuation of higher partial wave except S-wave. The numerical calculation indicates that the emission rate generally decreases with increase of mass.

Unruh has shown in his paper [16] that the S-wave cross section of Dirac fermion is exactly $1 / 8$ of that of scalar in the low-energy limit. It is interesting to apply our numerical method to check whether or not this ratio is maintained in the full range of energy. In Ref. [20] we examined the effect of mass in the absorption problem of the higher-dimensional object such as $p$-brane. Since, however, the low-energy of the scalar field is assumed from the beginning for the analytic computation, it was hard to understand the high-energy behavior. Recently, the absorption and emission problems of higher-dimensional black hole for massless particles with spin was numerically investigated in Ref. [34,35] in the full range of energy. In this 
context it seems to be interesting to apply our method for the investigation of mass effect in the problem of higher-dimensional objects.

Acknowledgement: This work was supported by the Korea Research Foundation Grant (KRF-2003-015-C00109). 


\section{REFERENCES}

[1] J. D. Bekenstein, Black Holes and Entropy, Phys. Rev. D7 (1973) 2333; Generalized second law of thermodynamics in black-hole physics, Phys. Rev. D9 (1974) 3292; Statistical black-hole thermodynamics, Phys. Rev. D12 (1975) 3077.

[2] S. W. Hawking, Black holes and thermodynamics, Phys. Rev. D13 (1976) 191.

[3] A. Strominger and C. Vafa, Microscopic origin of the Bekenstein-Hawking entropy, Phys. Lett. B379 (1996) 99 [hep-th/9601029].

[4] A. W. Peet, TASI Lectures on Black Holes in String Theory [hep-th/0008241] and references therein.

[5] S. W. Hawking, Black hole explosions?, Nature 248 (1974) 30.

[6] S. W. Hawking, Particle Creation by Black Holes, Commun. Math. Phys. 43 (1975) 199.

[7] N. Sanchez, Wave scattering and the absorption problem for a black hole, Phys. Rev. D16 (1977) 937.

[8] S. W. Hawking, Breakdown of Predictability in gravitational collapse, Phys. Rev. D14 (1976) 2460.

[9] J. Maldacena, D-branes and Near Extremal Black Holes at Low Energies, Phys. Rev. D55 (1997) 7645 [hep-th/9611125].

[10] G. T. Horowitz and J. Maldacena, The Black Hole Final State, JHEP 0402 (2004) 008 [hep-th/0310281].

[11] J. Maldacena and A. Strominger, Black Hole Greybody Factors and D-Brane Spectroscopy, Phys. Rev. D55 (1997) 861 [hep-th/9609026].

[12] A. A. Starobinsky, Amplification of waves during reflection from a rotating black hole, Zh. Eksp. Teor. Fiz. 64 (1973) 48 [Sov. Phys. - JETP 37 (1973) 28]. 
[13] B. Carter, Charge and Particle Conservation in Black-Hole Decay, Phys. Rev. Lett. 33 (1974) 558 .

[14] L. H. Ford, Quantization of a scalar field in the Kerr spacetime, Phys. Rev. D12 (1975) 2963.

[15] D. N. Page, Particle emission rate from a black hole: Massless particles from an uncharged, nonrotating hole, Phys. Rev. D13 (1976) 198.

[16] W. G. Unruh, Absorption cross section of small black holes, Phys. Rev. D14 (1976) 3251.

[17] S. R. Das, G. Gibbons, and S. D. Mathur, Universality of Low Energy Absorption Cross Sections for Black Holes, Phys. Rev. Lett. 78 (1997) 417 [hep-th/9609052].

[18] R. Emparan, Absorption of Scalars by Extended Objects, Nucl. Phys. B516 (1998) 297 [hep-th/9706204].

[19] D. K. Park and H. J. W. Müller-Kirsten, Universality or Non-Universality of Absorption Cross Sections for Extended Objects, Phys. Lett. B492 (2000) 135 [hep-th/0008215].

[20] E. Jung, S. H. Kim, and D. K. Park, Absorption Cross Section for S-wave massive Scalar, Phys. Lett. B586 (2004) 390 [hep-th/0311036].

[21] C. G. Callan and J. M. Maldacena, Brane dynamics from the Born-Infeld action, Nucl. Phys. B513 (1998) 198 [hep-th/9708147].

[22] G. W. Gibbons, Born-Infeld Particles and Dirichlet p-branes, Nucl. Phys. B514 (1998) 603 [hep-th/9709027].

[23] S. S. Gubser and A. Hashimoto, Exact absorption probabilities for the D3-brane, Commun. Math. Phys. 208 (1999) 325 [hep-th/9805140].

[24] M. Cvetic, H. Lü, and J. F. Vazquez-Poritz, Massive-scalar absorption by extremal pbranes, Phys. Lett. B462 (1999) 62 [hep-th/9904135]. 
[25] R. Manvelyan, H. J. W. Müller-Kirsten, J. -Q. Liang and Y. Zhang, Absorption Cross Section of Scalar Field in Supergravity Background, Nucl. Phys. B579 (2000) 177 [hepth/0001179].

[26] M. Cvetic, H. Lü, and J. F. Vazquez-Poritz, Absorption by Extremal D3-brane, JHEP 0102 (2001) 012 [hep-th/0002128].

[27] D. K. Park, S. N. Tamaryan, H. J. W. Müller-Kirsten, and J. Zhang, D-Branes and their Absorptivity in Born-Infeld Theory, Nucl. Phys. B594 (2001) 243 [hep-th/0005165].

[28] N. Sanchez, Absorption and emission spectra of a Schwarzschild black hole, Phys. Rev. D18 (1978) 1030.

[29] N. Sanchez, in String Theory in Curved Space Time, ed. N. Scnchez (World Scientific, Singapore, 1998).

[30] S. Persides, On the radial wave equation in Schwarzschild's spacetime, J. Math. Phys. 14 (1973) 1017.

[31] S. Persides, Global Properties of Radial Wave Functions in Schwarzschild's Space-Time: The Regular Singular Points, Commun. Math. Phys. 48 (1976) 165.

[32] S. Persides, Global Properties of Radial Wave Functions in Schwarzschild's Space-Time: The Irregular Singular Point, Commun. Math. Phys. 50 (1976) 229.

[33] R. Jackiw, in Lectures on Current Algebra and Its Application, ed. S. B. Treiman, R. Jackiw, and D. J. Gross (Princeton University Press, 1972, Princeton).

[34] P. Kanti and J. March-Russell, Calculable corrections to brane black hole decay: The scalar case, Phys. Rev. D66 (2002) 024023 [hep-ph/0203223].

[35] C. M. Harris and P. Kanti, Hawking Radiation for a $(4+n)$-dimensional Black Hole: Exact Results for the Schwarzschild Phase, JHEP 0310 (2003) 014 [hep-ph/0309054]. 


\section{Appendix}

In this Appendix we will show Eq.(3.5) explicitly. Since $\mathcal{F}_{\ell( \pm)}\left(x, x_{s}\right)$ are solutions, they should satisfy Eq.(3.1) separately. Multiplying $\mathcal{F}_{\ell( \pm)}$ to the equations of $\mathcal{F}_{\ell(\mp)}$ and subtracting them subsequently, one can derive easily

$$
\frac{d W}{d x}+\frac{2 x-x_{s}}{x\left(x-x_{s}\right)} W=0
$$

where $W$ is the Wronskian defined in Eq.(3.5). Solving (A.1) easily gives

$$
W=\frac{\mathcal{C}}{x\left(x-x_{s}\right)}
$$

where $\mathcal{C}$ is an integration constant.

In order to compute $\mathcal{C}$ we use the explicit expressions of $\mathcal{F}_{\ell( \pm)}$

$$
\begin{aligned}
& \mathcal{F}_{\ell(+)}\left(x, x_{s}\right)=i^{\ell+1} e^{-i\left[x+\frac{x_{s}}{v} \ln \left|x-x_{s}\right|\right]}\left(\frac{1}{x}+\cdots\right) \\
& \mathcal{F}_{\ell(-)}\left(x, x_{s}\right)=(-i)^{\ell+1} e^{i\left[x+\frac{x_{s}}{v} \ln \left|x-x_{s}\right|\right]}\left(\frac{1}{x}+\cdots\right) .
\end{aligned}
$$

Then the explicit computation of $W$ with these expressions yields the following leading term

$$
W \sim \frac{2 i}{x^{2}} \sim \frac{2 i}{x\left(x-x_{s}\right)}
$$

where the last approximation comes from the fact that $\mathcal{F}_{\ell( \pm)}$ is an asymptotic solutions. Thus we have $\mathcal{C}=2 i$.

Same procedure can be applied to $\mathcal{G}_{\ell}^{*}\left(x, x_{s}\right)$ and $\mathcal{G}_{\ell}\left(x, x_{s}\right)$, which yields $\mathcal{C}=-2 i\left|g_{\ell}\right|^{2} x_{s}^{2} / v$. 


\section{FIGURES}

FIG. 1. Plot of the partial absorption cross sections for massless scalar case. The black lines represent the partial absorption cross sections computed by analytic solutions and their analytic continuations. The red lines are result of Ref.[16]. The fact $\sigma_{0}=4 \pi$ at $k=0$ indicates the universality of the low-energy absorption cross section for S-wave. The peak points of $\sigma_{\ell}$ are given in Table I.

FIG. 2. The $r_{*}$-dependence of the effective potential (2.4) for massless case. The potential makes a barrier, which separates the asymptotic and near-horizon regions. The barrier heights are given in Table I.

FIG. 3. The energy-dependence of the total absorption cross sections when $m=0,0.15,0.3$, and 0.5. The wiggly behavior indicates that each partial absorption cross section has a peak at different energy scale. Regardless of mass the total absorption cross sections approach to $27 \pi / 4$ at high-energy limit, which reflects the approximate conformal symmetry.

FIG. 4. The energy-dependence of the total emission rate when $m=0,0.15,0.3$, and 0.5 . The disappearance of the wiggly pattern indicates that the rapidly decreasing Planck factor suppresses the contribution of the higher partial waves. The scalar mass generally reduces the emission rate.

FIG. 5. The $r_{*}$-dependence of the effective potential (3.2) for $\ell=0$ (Fig. 5a), $\ell=1$ (Fig. 5b) and $\ell=2$ (Fig. 5c). The scalar mass generally lowers the barrier height and makes the near-horizon regime more stable than the asymptotic regime. When $m$ is larger than the critical mass $m_{c}$, the potential peak becomes negative and cannot play a role of barrier any more.

FIG. 6. The energy-dependence of the imaginary part of the phase shift for $\ell=0$ (Fig. 6a), $\ell=1$ (Fig. $6 \mathrm{~b}$ ) and $\ell=2$ (Fig. 6c). As in the case of massless scalar $\beta_{\ell}$ is a monotonically increasing function. However, the scalar mass generally decreases the increasing rate of $\beta_{\ell}$. 
FIG. 7. The energy-dependence of the partial absorption cross section for $\ell=0$ (Fig. 7a), $\ell=1$ (Fig. 7b) and $\ell=2$ (Fig. 7c). The scalar mass makes a local minimum in $\sigma_{0}$ when $m$ is less than the critical mass. If $m>m_{c}$, the partial absorption cross sections become monotonically decreasing functions regardless of $\ell$.

FIG. 8. The mass-dependence of the low-energy absorption cross section for S-wave. The increasing behavior of $\sigma_{0}$ indicates that the universality is generally broken when the scalar field has a mass. The red line is a result of Ref.[16]. 


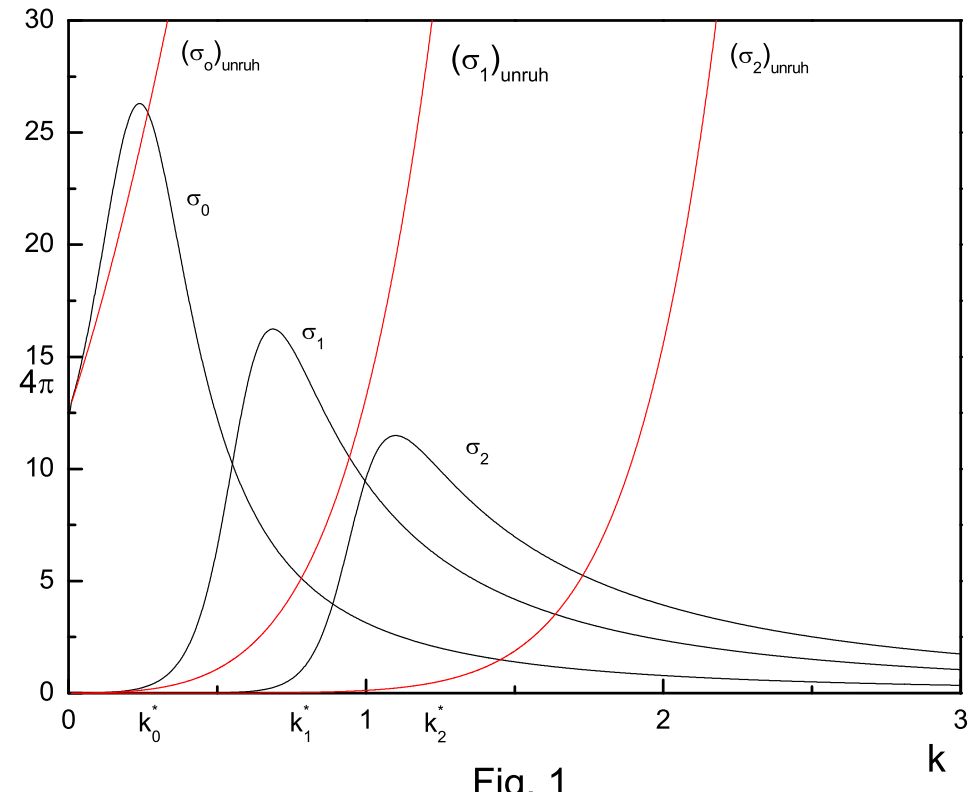

Fig. 1 


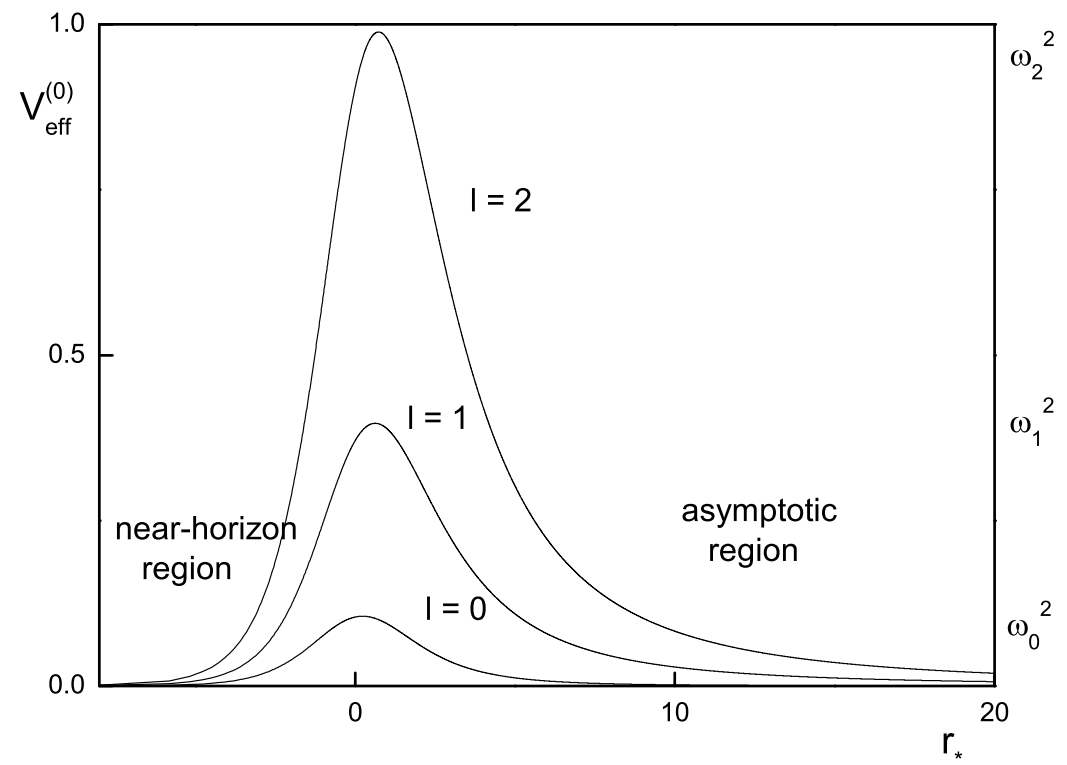

Fig. 2 


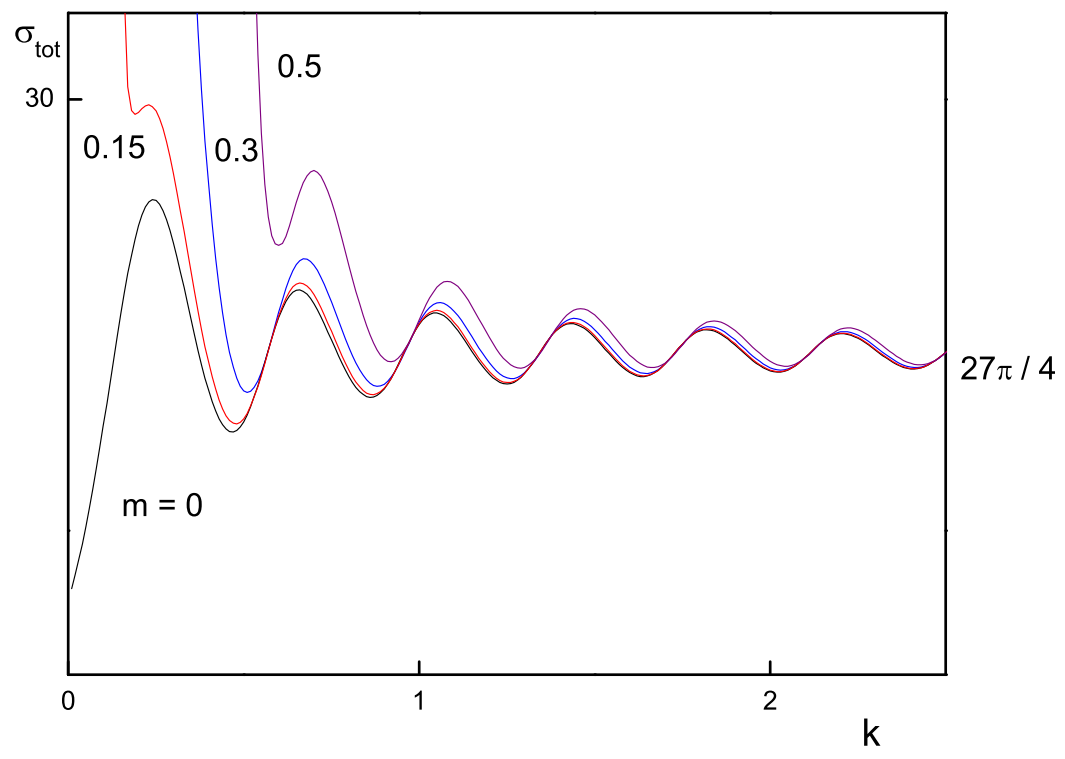

Fig. 3 


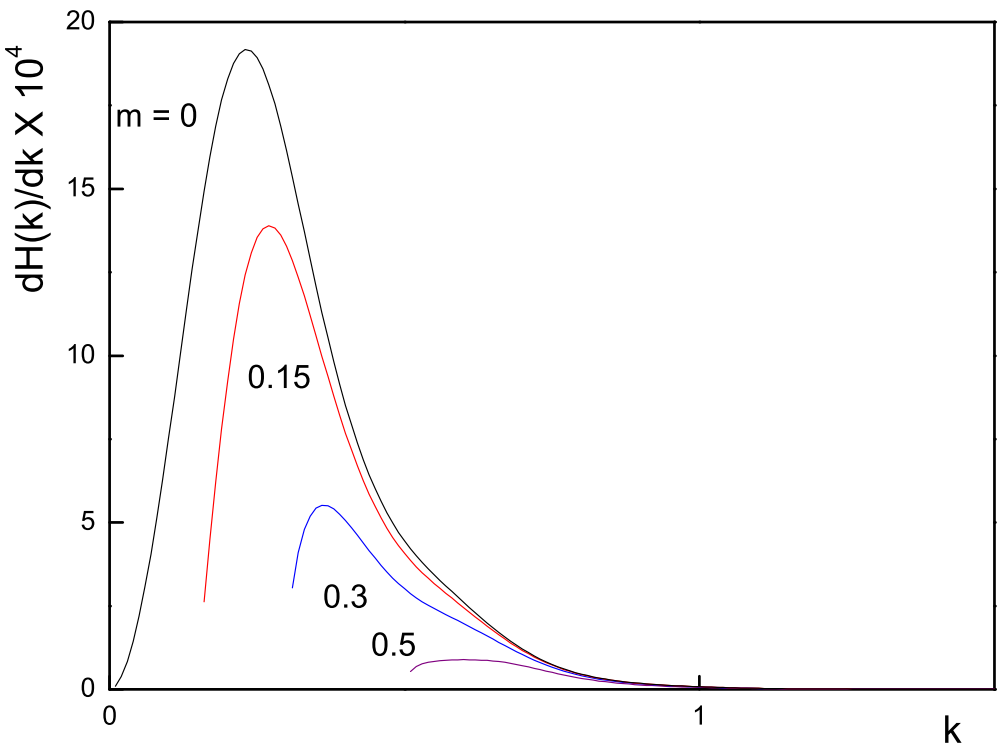

Fig. 4 


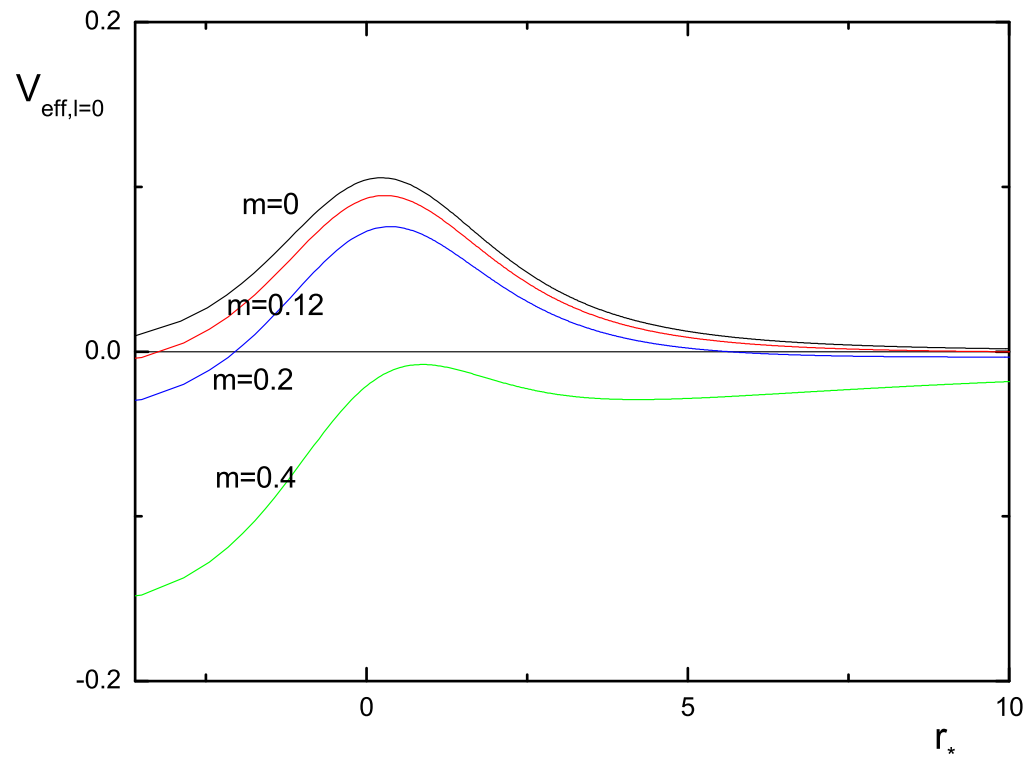

Fig. 5a 


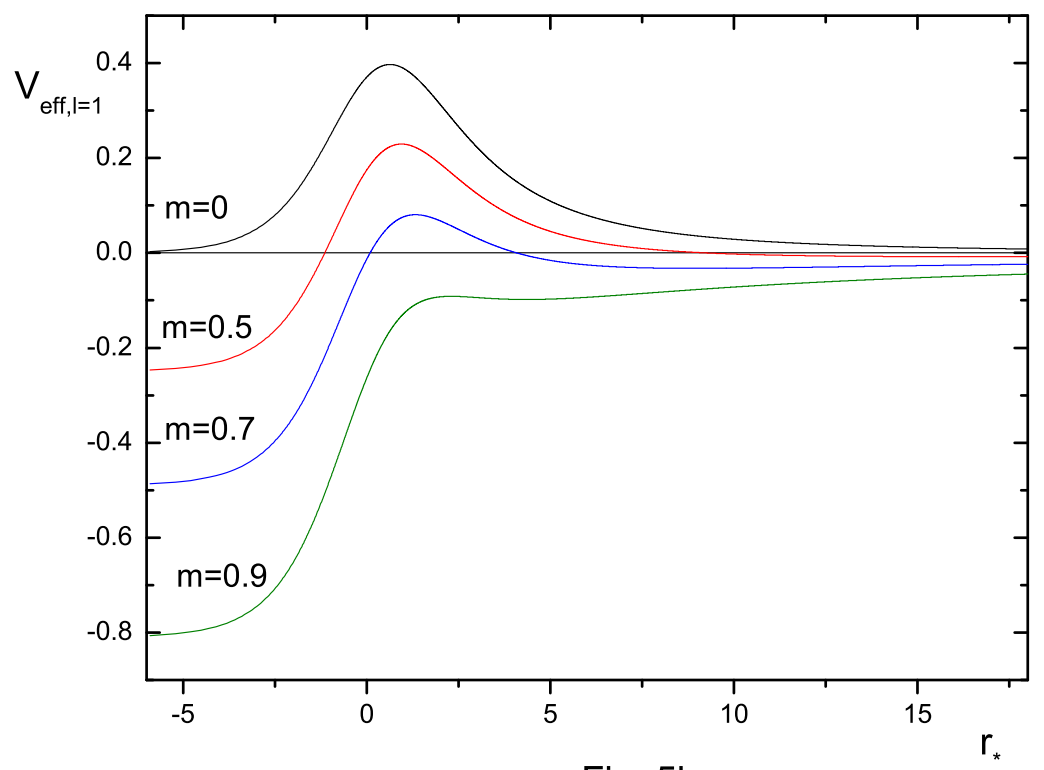

Fig. 5b 


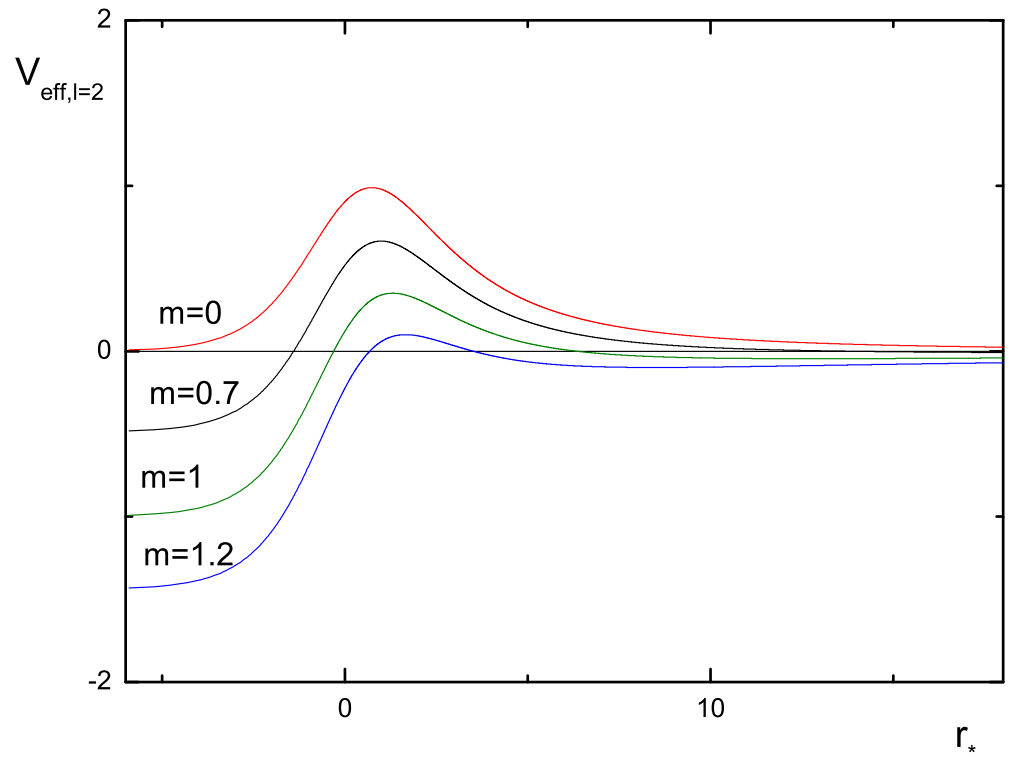

Fig. 5c 


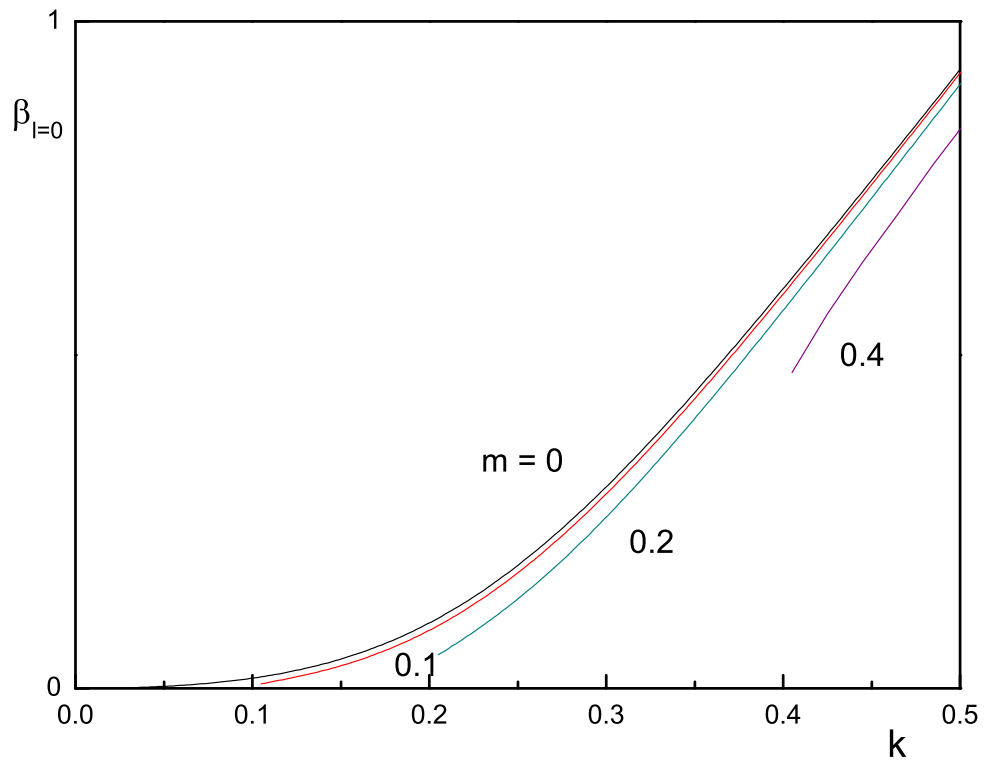

Fig. $6 a$ 


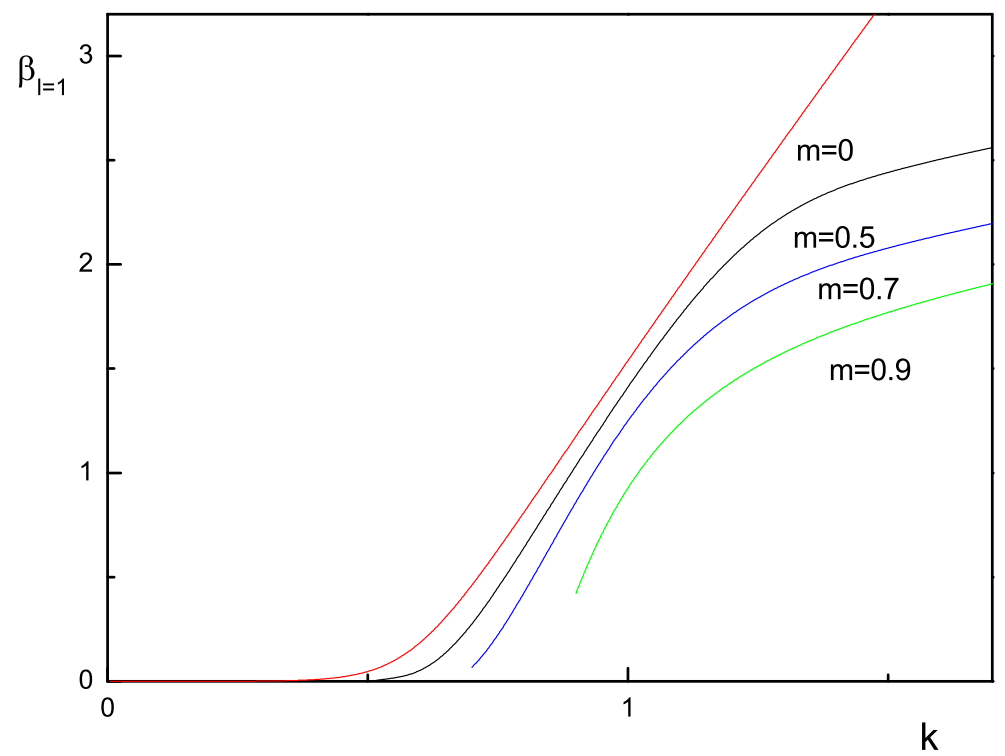

Fig. 6b 


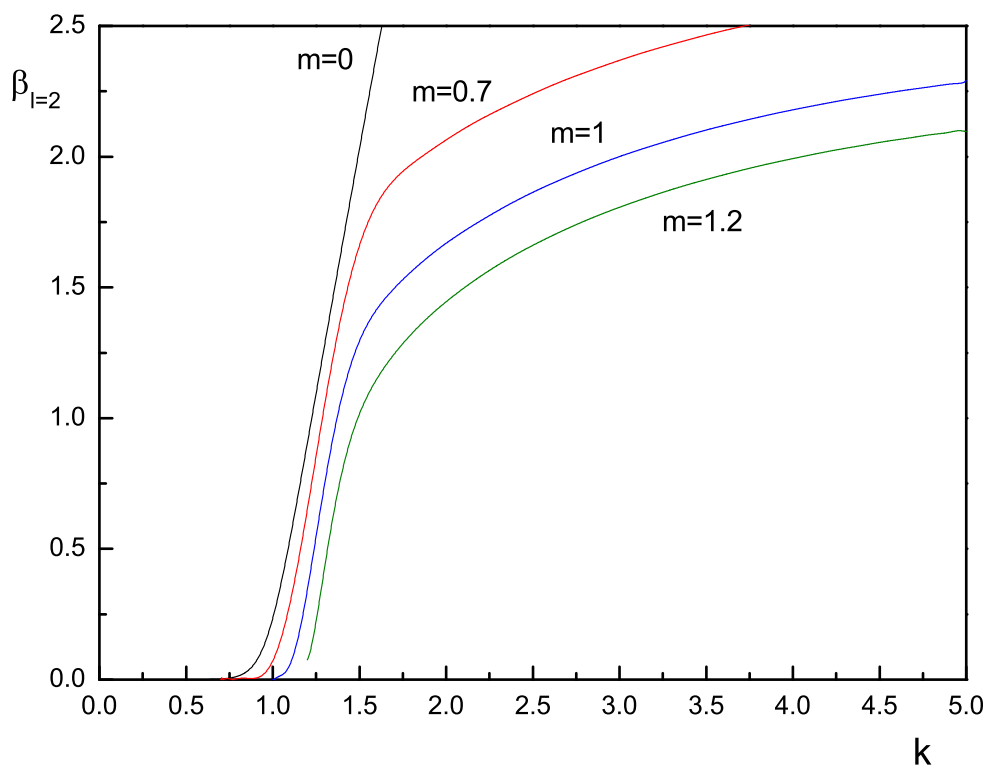

Fig. 6c 


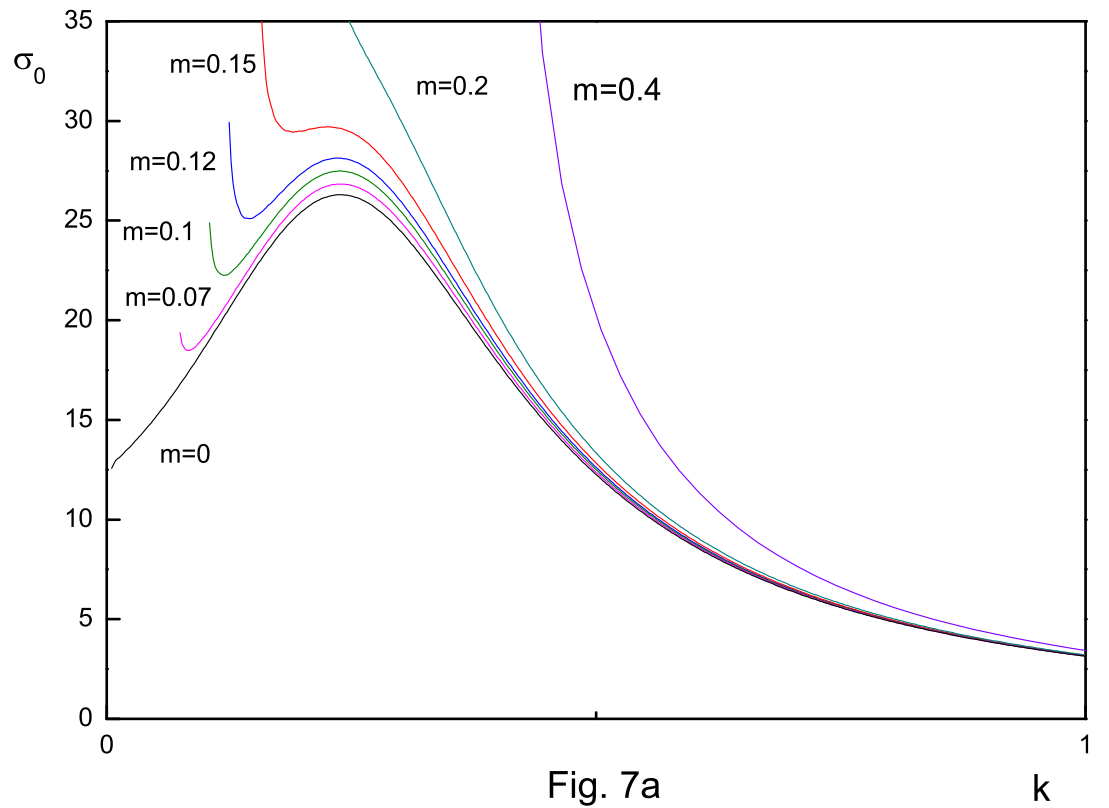




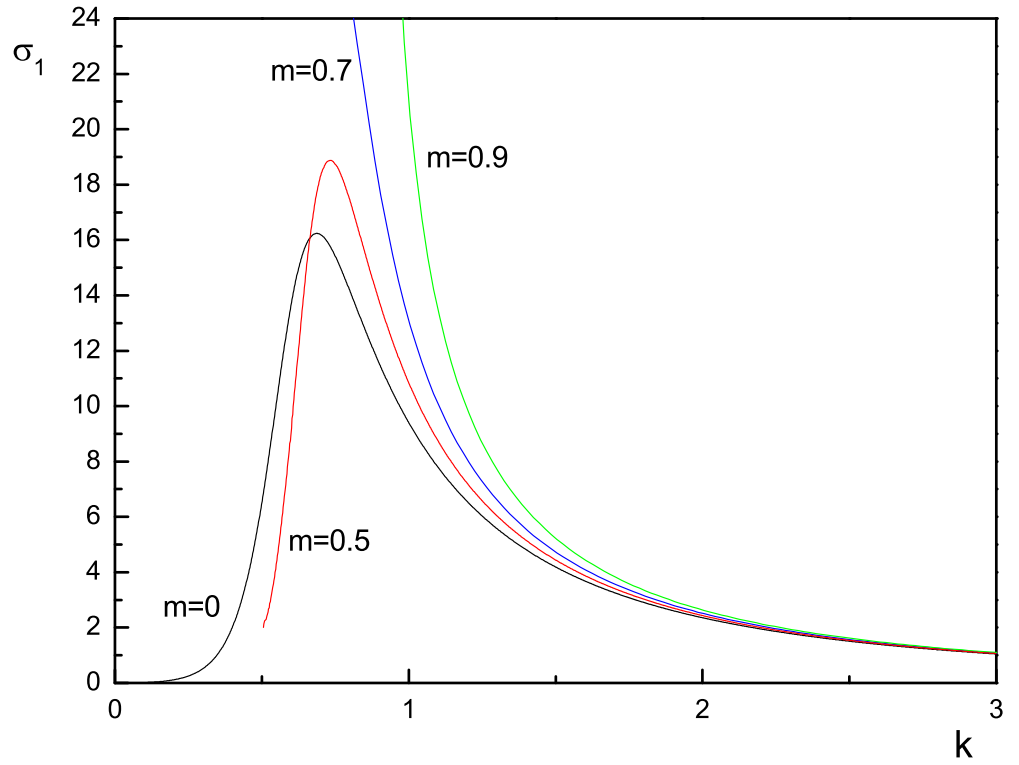

Fig. 7b 


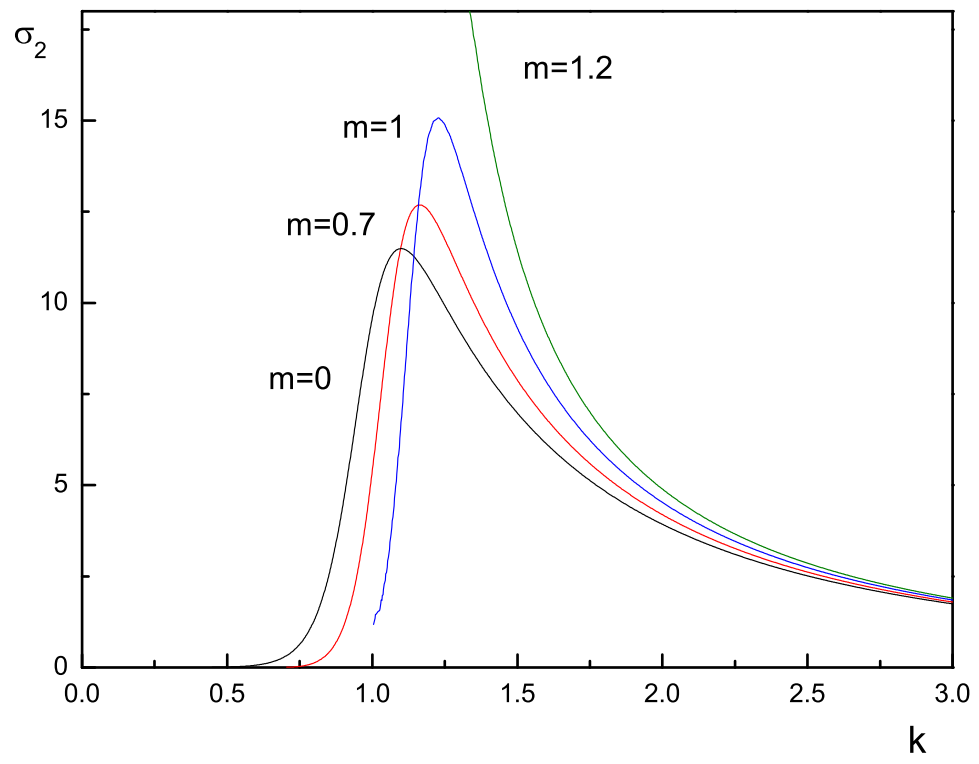

Fig. 7c 


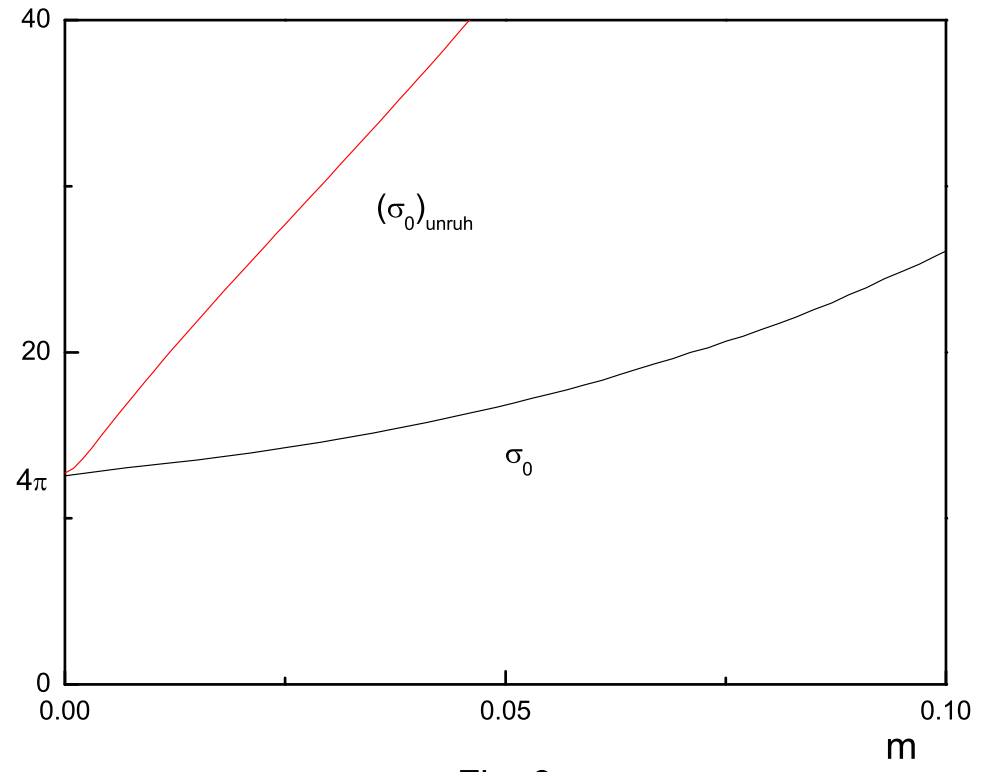

Fig. 8 